\title{
The assessment of cortisol using salivary ultrafiltrate
}

\author{
MARK A. LUMLEY \\ Wayne State University, Detroit, Michigan \\ WILLFRIED SCHRAMM \\ Saliva Diagnostic Systems, Vancouver, Washington \\ CYNTHIA S. POMERLEAU and OVIDE F. POMERLEAU \\ University of Michigan, Ann Arbor, Michigan \\ and \\ RICHARD H. SMITH \\ BioQuant Incorporated, Ann Arbor, Michigan
}

\begin{abstract}
Cortisol can be used to indicate stress level as well as to monitor certain disease states. Although cortisol can be sampled from blood and urine, saliva sampling has decided advantages. Unfortunately, whole saliva contains a number of substances that can metabolize or bind with cortisol, potentially confounding analysis and quantitation. We introduce a semipermeable pouch that accumulates a contaminant-free salivary ultrafiltrate, thereby overcoming a number of the problems encountered with whole saliva. In three studies, we demonstrate accuracy and utility of the device for cortisol determination: (1) in an artificial medium, $60 \%-77 \%$ of the cortisol was recovered in the ultrafiltrate; (2) in vivo, ultrafiltrate cortisol correlated highly with whole-saliva cortisol collected under ideal conditions; and (3) ultrafiltrate cortisol evinced positive relationships with depression and cigarette use, consistent with studies in the literature. We conclude that this device and salivafiltering technologies in general are useful in applications requiring quantitation of cortisol.
\end{abstract}

Behavioral researchers increasingly are quantitating biologically active molecules such as hormones, drugs and their metabolites, and immune markers. The steroid hormone cortisol has been the subject of much inquiry. Cortisol is an end product of hypothalamic-pituitaryadrenal (HPA) axis activity; it is released from the adrenal cortex following stimulation by pituitary adrenocorticotropic hormone (ACTH), regulated in turn by hypothalamic corticotropin releasing factor (CRF). Cortisol is relevant to psychosocial research because the HPA axis plays a major role in the stress response. Cortisol provides an objective marker for stress because it increases reliably and linearly (within certain limits) in response to a wide range of physical and psychosocial stressors, ranging from laboratory stress to dominance hierarchies among primates (Levine, Coe, \& Wiener, 1989; Linden, Dadgar, \& Earle, 1994; Munck, Guyre, \& Holbrook, 1984; Sapolsky, 1990).

Support for this research was provided in part by Grants DA 05982 and DA 06529 from the National Institute on Drug Abuse to the second and fourth authors, respectively. C. S. Pomerleau and O. F. Pomerleau are associated with the Behavioral Medicine Program, Department of Psychiatry, at the University of Michigan. Address correspondence and reprint requests to W. Schramm, Saliva Diagnostic Systems, 11719 N.E. 95th St., Vancouver, WA 98682.
Cortisol can be used as a marker of various disease states and health processes. Under certain conditions, cortisol decreases immunosurveillance, thus linking stress with infectious disease and cancer (Cohen \& Williamson, 1991; Meuleman \& Katz, 1985). Disregulation of the HPA axis occurs in depression, and a lack of cortisol suppression following administration of the synthetic steroid, dexamethasone, has both diagnostic and treatment implications (Arana \& Mossman, 1988; Ribeiro, Tandon, Grunhaus, \& Greden, 1993). Excessive cortisol levels may impair certain cognitive functions (Wolkowitz et al., 1990), and cortisol may alter body fat distribution and adversely affect cardiovascular health (Rodin, 1992). Cortisol levels increase in response to nicotine administration, which may influence nicotine sensitivity and subsequent cigarette smoking (Pomerleau \& Pomerleau, 1990a, 1990b).

Reliable and efficient quantitation of cortisol is useful. Although blood has been the traditional medium for cortisol assessment, it poses several disadvantages. Venipuncture must be conducted, requiring trained technicians. Venipuncture discourages some subjects from participating, elicits fear and/or fainting in others, and renders research with children, unsupervised field studies, and multiple assessments across time difficult or impossible. Repeated blood sampling for cortisol is particularly problematic, because the pain and/or stress of venipuncture 
elicits temporary cortisol elevations, necessitating prolonged adaptation periods. Finally, $90 \%$ to $95 \%$ of cortisol in the blood is reversibly bound to corticosteroidbinding globulin (CBG) proteins; although only the free (unbound) fraction of cortisol is physiologically active, most assays quantitate the total cortisol concentration. Urine samples also have been employed. Urine's principle advantage is the ability to accumulate cortisol secretion over an extended period; among the disadvantages are sampling and esthetics as well as the inability to pinpoint episodic stimulation of cortisol.

Saliva is an alternative to blood and urine sampling because many blood-borne molecules readily enter saliva via intercellular diffusion through the lipid bilayer of the acinar cells of the salivary gland. Only the free fraction of steroids such as cortisol diffuses through the cells, and concentrations are independent of salivary flow rate; thus, saliva concentrations are directly proportional tothough significantly lower than-plasma concentrations. The validity of saliva determination for a range of substances, including cortisol, is well recognized (Ferguson, 1984; Schramm, Kidwell, Smith, \& Craig, 1992; Tenovuo, 1989; Vining \& McGinley, 1986, 1987). Additionally, saliva collection obviates the many logistic and measurement reliability problems associated with venipuncture and blood sampling. For these reasons, salivary assessment of cortisol has been advocated as the preferred method (Kirschbaum \& Hellhammer, 1989).

Saliva assessment of cortisol poses unique problems, however. The usual saliva-sampling method requires repeated expectoration until sufficient whole saliva has been accumulated. Some subjects are disturbed by "spitting" into a tube and viewing their saliva; others experience "dry mouth." The time required to accumulate sufficient saliva varies among subjects, from several seconds to many minutes. Techniques sometimes employed by investigators to overcome dry mouth, such as chewing on an object such as parafilm or a rubber band, or even imagining biting a lemon, have unknown effects on cortisol secretion. Finally, expectoration requires motor activity by the subject, thus interrupting the ongoing stream of behavior.

More important, perhaps, is that whole saliva contains many components that may affect saliva processing and analysis. Oral squames, mucopolysaccharides, and mucoproteins contribute to saliva's highly viscous, sticky consistency, making laboratory handling (centrifugation and pipetting) laborious and inaccurate. Whole saliva contains substances that may affect the measured concentration of cortisol. Enzymes from food, oral microorganisms, or endothelium cells from the salivary gland may metabolize steroids (Laudat et al., 1988; McCracken \& Poland, 1989; Meulenberg \& Hofman, 1990). Although cooling or freezing of the saliva sample may slow or halt enzymatic metabolism, in practice, whole saliva often is subjected to enzymatic degradation because it is left at room temperature for extended and variable periods. Other saliva components can bind cortisol, resulting in erroneous estimates of the free fraction. For example, red blood cells and other blood proteins, which are found in $20 \%-50 \%$ of saliva samples (Piazza et al., 1989), may bind cortisol (Hiramatsu \& Nisula, 1988), as may food protein residues and gingival and mucosal exudates, which increase after toothbrushing (Schramm, Smith, Craig, \& Grates, 1993).

It is desirable to improve saliva collection procedures and minimize sources of processing difficulty and measurement error. A number of technologies have been developed to accomplish these goals, and it is the objective of this paper to introduce to a behavioral audience an ultrafiltrate device-an osmotic collector that consists of a small pouch made from a semipermeable membrane. The pouch is held in the mouth and collects an ultrafiltrate of saliva that is free of components that interfere with ease of handling and accurate cortisol measurement (Schramm, Annesley, Siegel, Sackellares, \& Smith, 1991). Supporting data are presented from three studies: two evaluate reliability and accuracy of the device in collecting cortisol from an artificial medium and from human saliva; the third demonstrates the device's utility to predict individual differences in depression and smoking. In the Discussion section below, saliva-filtering technologies will be examined and compared.

\section{STUDY 1 Accuracy of the Device In Vitro}

The first study was conducted using an artificial saliva medium to determine (1) the rate and extent of cortisol transfer into the osmotic collector, and (2) the degree of binding of cortisol to the device's external surface.

\section{Method}

Ultrafiltrate collection devices. The saliva collection devices were made from semipermeable membranes (regenerated cellulose) designed for hemodialysis (Cuprophan; Enka AG, Germany). Two membrane disks ( $35 \mathrm{~mm}$ in diameter and $20 \mu \mathrm{m}$ thick) were bonded with polyurethane (Tycel; Lord Corporation, Erie, PA) by applying a 5-mm-wide adhesive ring at the periphery and pressing the disks together. The membrane pores have an average molecular cutoff of 12,000 daltons, preventing entry into the pouch of relatively large molecules such as enzymes, mucopolysaccharides, and blood proteins, but allowing cortisol and other small molecules to pass through. Salivary flow is stimulated by two means: the pouch contains granular sucrose $(0.75 \mathrm{~g})$, which elicits salivation, and the pouch is moved around with the tongue. The pouch accumulates a volume of $1-1.5 \mathrm{ml}$ of salivary ultrafiltrate via osmosis. Because large molecules are excluded, the ultrafiltrate has low viscosity (less than that of serum) and can be processed without centrifugation (Schramm et al., 1991).

Reagents. Human serum albumin, alpha-amylase (type VIII-A from barley malt), lysozyme (grade III), mucin (type I), and cortisol were purchased from the Sigma Chemical Co., St. Louis. Human gamma globulin (IgG) and scintillation cocktail, CytoScint ES, were purchased from ICN Biomedicals, Irvine, CA. Radiolabeled ${ }^{3} \mathrm{H}$-cortisol $\left(\left[1,2-{ }^{3} \mathrm{H}(\mathrm{N})\right]\right.$-hydrocortisone, specific activity: $49.1 \mathrm{KCi} / \mathrm{mol}$ ) was obtained from NEN Research Products, DuPont Corp., Wilmington, DE. 


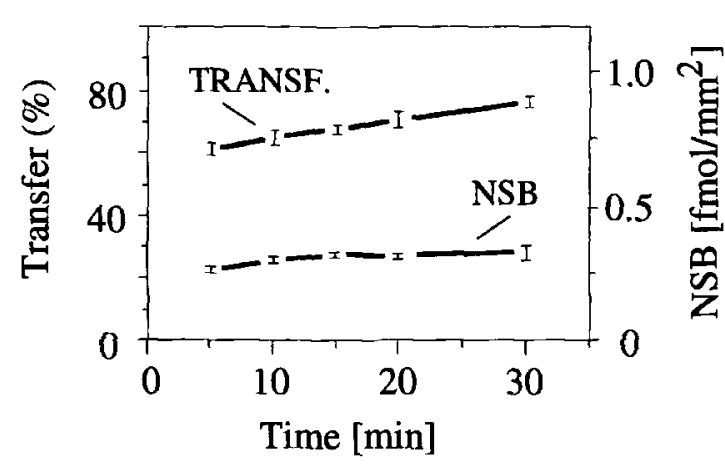

Figure 1. The transfer of cortisol into the collector, and nonspecific binding (NSB) of cortisol to the collector membrane during $\mathbf{3 0} \mathrm{min}$ of exposure to an artificial saliva medium.

Preparation of artificial saliva medium. We prepared a medium approximating the protein and electrolyte content of saliva: amylase $0.6 \mathrm{~g} / \mathrm{L}$; lysozyme $0.2 \mathrm{~g} / \mathrm{L} ;$ mucin $0.7 \mathrm{~g} / \mathrm{L} ; \operatorname{IgG} 0.2 \mathrm{~g} / \mathrm{L}$; albumin $75 \mathrm{mg} / \mathrm{L}$; inorganic phosphate $2.5 \mathrm{mmol} / \mathrm{L} ; \mathrm{Na}^{+} 60 \mathrm{mmol} / \mathrm{L}$; $\mathrm{K}^{+} 18 \mathrm{mmol} / \mathrm{L}$; urea $5 \mathrm{mmol} / \mathrm{L} ; \mathrm{I}^{-} 1.5 \mathrm{mmol} / \mathrm{L} ; \mathrm{Cl}^{-} 50 \mathrm{mmol} / \mathrm{L}$; pH 7.8. As a bacteriostat, $0.2 \mathrm{~g}$ of thimerosal per liter of solution was added.

Transfer of cortisol. Five polypropylene bags containing artificial saliva were equilibrated overnight. This solution was discarded and the bags were filled with $50 \mathrm{ml}$ of artificial saliva containing $1.5 \mathrm{ng} / \mathrm{ml}$ of cortisol and $127 \mathrm{pg} / \mathrm{ml}$ of ${ }^{3} \mathrm{H}$-cortisol $(23,000$ $\mathrm{cpm} / \mathrm{ml}$ ). The temperature of the solution was maintained at $37^{\circ} \mathrm{C}$ (body temperature). Three ultrafiltrate devices were added to each bag, and the bags were gently massaged by hand in a water bath at $37^{\circ} \mathrm{C}$ for varying periods of time $(5,10,15,20$, and $30 \mathrm{~min})$. After each time period, one bag was opened and the cortisol in the ultrafiltrate and in the external solution was measured by means of $\beta$-radiation of ${ }^{3} \mathrm{H}$-cortisol.

Nonspecific binding. To determine the extent of cortisol binding to the membranes of the device, the membranes used in this experiment were blotted on absorbent paper and immersed in $10 \mathrm{ml}$ of scintillation cocktail. Cortisol taken up by the membrane was measured by means of $\beta$-radiation of ${ }^{3} \mathrm{H}$-cortisol. Correction for quenching by the membrane material was not required. In prior experiments, no appreciable quenching of $\beta$-radiation from a known amount of ${ }^{3} \mathrm{H}$-cortisol was found in the presence of the membrane of the device.

\section{Results}

The transfer of cortisol from the external medium into the collector increased from about $60 \%$ after 5 min to about $77 \%$ after 30 min (Figure 1). At approximately $8 \mathrm{~min}$ of incubation, there was about $65 \%$ transfer. The nonspecific binding of cortisol to the semipermeable membrane was approximately $0.3 \mathrm{fmol} / \mathrm{mm}^{2}$ and changed little over time. Because the total surface area of the osmotic device is $1,900 \mathrm{~mm}^{2}$, about $0.2 \mathrm{ng}$ per device were nonspecifically bound.

\section{STUDY 2 Accuracy of the Device in Humans}

The second study was conducted in vivo to compare the accuracy of the saliva-filtering approach with whole saliva.

\section{Method}

Subjects. We collected samples from 5 healthy young adults (2 women, 3 men), who were habitual cigarette smokers (for at least 3 years) participating in a research study on abstinence from nicotine. The subjects were studied over 4 consecutive afternoons. They smoked as usual all day on Day 1 and then refrained from all nicotine on the subsequent 3 days.

Specimen collection. Whole-saliva and ultrafiltrate samples were collected from each subject during each of the first 3 days of the study; two pairs of samples were obtained on Day 4; thus, five sets of saliva specimens were accumulated for each subject, yielding 25 total sample pairs. For the collection, the device was moistened with tap water for $5 \mathrm{sec}$ and placed in the subject's mouth. The subject moved the device around with his/her tongue and held excess saliva in the mouth to permit absorption into the vehicle. After $8 \mathrm{~min}$, the subject expectorated excess whole saliva into a test tube, and also removed the device. The subject continued to spit into the tube until at least $2 \mathrm{ml}$ of whole saliva was collected. Both the ultrafiltration collectors and test tubes containing whole saliva were cooled immediately. Within $30 \mathrm{~min}$, the device was frozen to $-80^{\circ} \mathrm{C}$, and whole saliva in the tubes was centrifuged at $3,000 \mathrm{rpm}$ for $15 \mathrm{~min}$. At least $1 \mathrm{ml}$ of supernatant from whole saliva was separated and frozen for analysis.

Immunoassay. Cortisol in whole saliva and ultrafiltrate was quantitated by solid-phase radioinmunoassay (Diagnostic Products Corporation, Los Angeles) adapted for the measurement of cortisol in saliva according to the manufacturer's recommendations, with some modifications. The assay was performed in $12 \times$ $75 \mathrm{~mm}$ silicon-coated tubes with a total volume of $1.6 \mathrm{ml}$ and $200 \mu \mathrm{l}$ of sample volume. The sample volume was increased if required. A ${ }^{125}$-labeled cortisol derivative was used as tracer. From the logit-log expression of the dose-response curve, we measured $(M \pm S D)$ at $80 \%$ tracer inhibition, $0.147 \pm 0.012 \mu \mathrm{g} / \mathrm{ml}$; at $50 \%$, $0.75 \pm 0.05 \mu \mathrm{g} / \mathrm{ml}$; and at $20 \%, 3.8 \pm 0.2 \mu \mathrm{g} / \mathrm{ml}$.

Correction for partial molal volume. The ultrafiltrate of saliva contains sucrose that occupies a certain volume of pipetted ultrafiltrate. Therefore, the pipetted volume has to be corrected for the partial molal volume. The total water concentration in the ultrafiltrate, $C_{\mathrm{W}}(\mathrm{g} / \mathrm{m})$ ), is a function of the relative density, $D$. The water concentration and the density follow the equation $C_{\mathrm{W}}=$ $2.7-1.7 D$, from which the actual concentration, $C_{\mathrm{ACT}}$, can be calculated from the concentration measured in the ultrafiltrate, $C_{\mathrm{UF}}$, by the equation $C_{\mathrm{ACT}}=C_{\mathrm{UF}} / C_{\mathrm{W}}$. By weighing $1 \mathrm{ml}$ on an analytical balance, we determined the average density of saliva ultrafiltrate, yielding a mean density of $1.142 \pm 0.032(n=35)$. Thus, the average correction used for concentration was $C_{\mathrm{W}}=0.76$.

\section{Results}

Whole-saliva and ultrafiltrate cortisol concentrations followed a linear regression with a correlation coefficient of $r=.91$ (Figure 2). Two of the cortisol pairs from 2 subjects were identical and relatively high (see Figure 2), thus potentially overestimating the correlation; yet the correlation was only slightly reduced $(r=.84)$ when these pairs were excluded. When the correction for partial molal volume was applied to the ultrafiltrate concentrations, the cortisol concentration in the ultrafiltrate averaged $79 \%$ of that found in whole saliva.

\section{STUDY 3 Ultrafiltrate Cortisol and Individual Differences}

In this study, we evaluated whether subject differences in depression and cigarette use predicted ultrafiltrate cortisol levels. Both depression (Arana \& Mossman, 1988; 


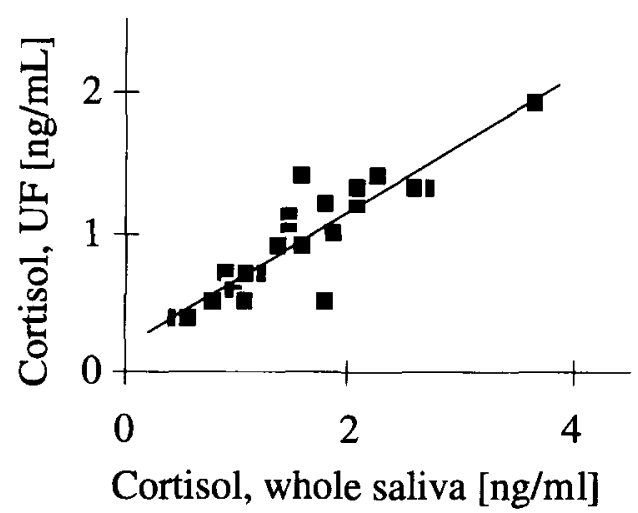

Figure 2. Cortisol concentrations in the ultrafiltrate (UF) as a function of cortisol concentrations in whole saliva from 5 human subjects. The regression equation is $C_{\mathrm{UF}}=0.26+0.61 C_{\mathrm{WHOLE}}$; standard error of estimate: $0.19 \mathrm{ng} / \mathrm{ml}$.

Kalin \& Dawson, 1986) and nicotine (Pomerleau \& Pomerleau, 1990b; Wilkins et al., 1982) are known to covary positively with cortisol.

\begin{abstract}
Method
Subjects and Procedures. Twenty 23- to 36-year-old adult smokers ( 10 women) were recruited from the local community to participate in the research project on abstinence from nicotine described under Study 2 above. On the first afternoon, the subjects came to the laboratory, smoked a single cigarette, and completed a depression questionnaire. An ult rafilt rate of saliva was collected with the device, as described in Study 2,45 min after the cigarette was smoked. The ultrafiltrate was stored and cortisol analyzed using the immunoassay and correction for partial molal volume as in Study 2.
\end{abstract}

Depression measure. Depression was assessed with the Center for Epidemiologic Studies-Depression Scale (CES-D; Radloff, 1977), an inventory of 20 symptoms, which are rated on a 4-point scale $(0-3)$ for the frequency that each symptom was experienced during the previous week. Possible scores range from 0 to 60 ; higher scores reflect increased depression. The CES-D is more sensitive to current than to long-standing depression, and it emphasizes affective and cognitive rather than vegetative symptoms. The instrument has been shown to have very high internal consistency and relatively high correlations with alternative questionnaire and interview measures of depression.

\section{Results}

Depression and daily cigarette frequency correlated significantly with ultrafiltrate cortisol (cortisol in $\mathrm{ng} / \mathrm{ml}$, $M=1.5, S D=0.6$, range $=0.6-2.8$ ). As hypothesized, greater depression (CES-D, $M=11.7, S D=9.1$, range = $0-31)$ correlated with higher cortisol levels $(r=.46$, $p=.04)$. Additionally, greater daily cigarette frequency $(M=23.5, S D=7.1$, range $=8-40)$ correlated with higher cortisol $(r=.64, p=.002)$. Depression and cigarette frequency were not significantly correlated $(r=$ .08 ), indicating that they were relatively independent predictors of cortisol.

\section{DISCUSSION}

This paper introduces to behavioral research a technology that facilitates the assessment of cortisol. The use of saliva obviates difficulties associated with urine and blood-based cortisol assessment, and the ultrafiltration technology appears to improve ease of collection and handling as well as accuracy. Subjects in this and other studies have reported that use of the device is more acceptable than repeated expectoration, and an ample supply of saliva is stimulated quickly, thus preventing "dry mouth." Furthermore, the collection procedures and duration are standardized and behaviorally passive, minimizing interruption of subjects' ongoing behavior. The device yields an ultrafiltrate free from viscous polysaccharides and mucoproteins - substances that make laboratory processing of saliva more difficult. Ultrafiltrate technology also has the potential to reduce measurement error by excluding blood components, food residues, enzymes, oral microorganisms, and other large particles that can confound the assay via cortisol binding or metabolism. Although samples should be refrigerated, brief storage at room temperature does not jeopardize the integrity of the specimen. Also, as suggested by its ability to assess the presence and amount of drugs such as cotinine (nicotine metabolite), phenytoin (anticonvulsant drug), and cocaine (Schramm et al., 1991; Schramm, Craig, Smith, \& Berger, 1993; Schramm, Pomerleau, Pomerleau, \& Grates, 1992), the device has potential clinical diagńostic value.

Cortisol collected using this technology appears to accurately reflect levels both in an artificial saliva medium and in whole human saliva. Approximately $75 \%$ of the cortisol in whole saliva transferred to the ultrafiltrate, and there was a very high correlation between cortisol concentrations in the ultrafiltrate and whole saliva. Utility of the device was demonstrated by replicating previous observations of individual differences in cortisol levels: subjects with greater depression scores and higher frequencies of daily cigarette use had higher levels of cortisol in the ultrafiltrate.

Clearly, further research on this technology is needed. The loss of $0.2 \mathrm{ng}$ per device due to nonspecific membrane binding may result in some underestimation of cortisol at low concentrations. Yet this amount $(0.2 \mathrm{ng})$ is minimal compared with a typical cortisol concentration of about $0.6-6 \mathrm{ng} / \mathrm{ml}$ in saliva, and considering that up to $10 \mathrm{ml}$ of saliva flow past the device during the collection period. Nonetheless, it would be desirable to reduce the membrane binding, perhaps by using other membrane materials, such as a copolymer of polycarbonate and polyethylene (Gambro $\mathrm{GmbH}$, Hechingen, Germany). The lower cortisol concentrations found in the ultrafiltrate than in whole saliva are probably due to hydrophilic/lipophilic interactions between cortisol and the membrane rather than to a prevention of cortisol 
entry because of the pore size. A correction of saliva concentrations would be needed if direct comparisons with whole saliva or blood were desired. Although the collection of cortisol into the device changes minimally over $30 \mathrm{~min}$ in a stable, artificial saliva medium, it would be desirable to examine the rate of accumulation over the 8 -min collection period used with humans. Although this technology appears to be appropriate for studies examining relatively stable or enduring individual or group differences in cortisol, further study is needed to confirm that the ultrafiltrate technology accurately assesses acute changes in cortisol as a function of physical, psychosocial, or pharmacological stimulation. It is expected that the device can assess short-term differences in cortisol levels, however, because it accurately reflects current whole-saliva cortisol concentrations, and whole saliva is sensitive to acute cortisol changes (Kirschbaum \& Hellhammer, 1989).

Several other technologies have been developed to circumvent the collection and measurement problems associated with whole saliva. The use of a cotton plug ("Salivette") in the buccal cavity to accumulate whole saliva obviates some of the collection problems (Hellhammer, Kirschbaum, \& Belkien, 1987). The large surface area binds some molecules, however, thereby limiting measurement accuracy, and the whole-saliva product still must be processed and is exposed to enzymes and other components that could increase measurement error (Schramm, Kidwell, et al., 1992; Vining \& McGinley, 1986).

A promising filtrate technology similar to the device presented here has been developed and tested by Wade and colleagues (Shipley, Alessi, Wade, Haegele, \& Helmbold, 1992; Wade, 1992a, 1992b; Wade \& Haegele, 1991). The "oral diffusion sink" (ODS) is a small device, worn unobtrusively in the mouth, that accumulates substances by binding them to a specific antiserum in the device. A dialysis membrane prevents entry of larger components. Unlike the ultrafiltrate collector, the ODS is designed to accumulate substances over a period of hours, however, yielding an integrated value that is minimally affected by acute changes in cortisol levels. Wade and colleagues have demonstrated, in an elegant series of studies, the utility of the ODS for assessing cortisol and other steroids. Although both the ODS and the ultrafiltrate device filter out large substances and accumulate a medium suitable for the measurement of small molecules, the ultrafiltrate device is designed to measure the integrated concentration during brief collection periods ( $8 \mathrm{~min}$ ). Although research has not directly compared these two approaches, both devices show potential for simplifying and improving saliva sampling of cortisol along with other molecules that may be of interest to behavioral scientists.

\section{REFERENCES}

Arana, G. W., \& Mossman, D. (1988). The dexamethasone suppression test and depression. Endocrinology \& Metabolism Clinics of North America, 17, 21-39.
Cohen, S., \& Williamson, G. M. (1991). Stress and infectious disease in humans. Psychological Bulletin, 109, 5-24.

Ferguson, D. B. (1984). Steroid hormones in saliva. Munich: S. Karger.

Hellhammer, D. H., Kirschbaum, C., \& Belkien, L. (1987). Measurement of salivary cortisol under psychological stimulation. In J. N. Hingtgen, D. Hellhammer, \& G. Huppmann (Eds.), Advanced methods in psychobiology (pp. 281-289). Toronto: Hogrefe.

Hiramatsu, R., \& Nisula, B. C. (1988). Erythrocyte-associated component of blood cortisol. In R. Frairia, H. L. Bradlow, \& G. Gaidano (Eds.), Steroid-protein interactions: Basic and clinical aspects (Annals of the New York Academy of Sciences, Vol. 538, pp. 159-166). New York: New York Academy of Sciences.

KaliN, N. H., \& Dawson, G. W. (1986). Neuroendocrine dysfunction in depression: Hypothalamic-anterior pituitary systems. Trends in Neurosciences, 9, 261-266.

Kirschbaum, C., \& Hellhammer, D. H. (1989). Salivary cortisol in psychological research: An overview. Neuropsychobiology, 22, 150169.

Laudat, N. J., Cerdas, S., Fournier, C., Guiban, D., Guilhaume, B., \& LuTON, J. P. (1988). Salivary cortisol measurement: A practical approach to assess pituitary-adrenal function. Journal of Clinical Endocrinology \& Metabolism, 66, 343-348.

Levine, S., CoE, C., \& Wiener, S. (1989). The psychoneuroendocrinology of stress: A psychobiological perspective. In S. Levine \& R. Brush (Eds.), Psychoendocrinology (pp. 181-207). New York: Academic Press.

Linden, W., Dadgar, N., \& Earle, T. L. (1994, April). Cortisol responses to acute stress: $A$ selective review. Paper presented at the annual meeting of the American Psychosomatic Society, Boston.

McCracken, J. T., \& Poland, R. E. (1989). Saliva and serum dynamics following intravenous dexamethasone in normal volunteers. Life Sciences, 45, 1781-1785.

Meuleman, J., \& Katz, P. (1985). The immunologic effects, kinetics, and use of glucocorticosteroids. Medical Clinics of North America, 69, 805-816.

Meulenberg, P. M. M., \& Hofman, J. A. (1990). Differences between concentrations of salivary cortisol and cortisone and of free cortisol and cortisone in plasma during pregnancy and postpartum. Clinical Chemistry, 36, 70-75.

Munck, A., Guyre, P. M., \& Holbrook, N. J. (1984). Physiological functions of glucocorticoids in stress and their relation to pharmacological actions. Endocrine Reviews, 5, 25-44.

Piazza, M., Chirianni, A., Picciotto, L., Guadagnino, V., OrLANDo, R., \& Cataldo, P. T. (1989). Passionate kissing and microlesions of the oral mucosa: Possible role in AIDS transmission. Journal of the American Medical Association, 261, 244-245.

Pomerleau, O. F., \& Pomerleau, C. S. (1990a). Cortisol response to a psychological stressor and/or nicotine. Pharmacology, Biochemistry \& Behavior, 36, 211-213.

Pomerleau, O. F., \& Pomerleau, C. S. (1990b). Dexamethasone attenuation of the cortisol response to nicotine in smokers. Psychopharmacology, 101, 284-286.

RADLOFF, L. S. (1977). The CES-D scale: A self-report depression scale for research in the general population. Applied Psychological Measurement, 1, 385-401.

Ribeiro, S. C. M., Tandon, R., Grunhaus, L., \& Greden, J. F. (1993). The DST as a predictor of outcome in depression: A meta-analysis. American Journal of Psychiatry, 150, 1618-1629.

RoDin, J. (1992). Determinants of body fat localization and its implications for health. Annals of Behavioral Medicine, 14, 275-281.

SAPOLSKY, R. M. (1990). Adrenocortical function, social rank, and personality among wild baboons. Biological Psychiatry, 28, 862-878.

Schramm, W., ANnesley, T. M., Siegel, G. J., Sackellares, J. C., \& SMITH, R. H. (1991). Measurement of phenytoin and carbamazepine in an ult rafiltrate of saliva. Therapeutic Drug Monitoring, 13, 452460 .

Schramm, W., Craig, P. A., Smith, R. H., \& Berger, G. E. (1993). Cocaine and benzoylecgonine in saliva, serum, and urine. Clinical Chemistry, 39, 481-487.

Schramm, W., Kidwell, D. A., Smith, R. H., \& Craig, P. A. (1992). 
Drugs of abuse in saliva: A review. Journal of Analytical Toxicolology, 16, 1-9.

Schramm, W., Pomerleau, O. F., Pomerleau, C. S., \& Grates, H. E. (1992). Cotinine in an ultrafiltrate of saliva. Preventive Medicine, 21, 63-73.

Schramm, W., Smith, R. H., Craig, P. A., \& Grates, H. E. (1993). Testosterone concentration is increased in whole saliva, but not in ultrafiltrate, after toothbrushing. Clinical Chemistry, 39, 519-521.

Shipley, J. E., Alessi, N. E., Wade, S. E., Haegele, A. D., \& HelmBOLD, B. (1992). Utility of an oral diffusion sink (ODS) device for quantification of saliva corticosteriods in human subjects. Journal of Clinical Endocrinology \& Metabolism, 74, 698-700.

Tenovuo, J. O. (1989). Human saliva: Clinical chemistry and microbiology. Boca Raton, FL: CRC Press.

VINING, R. F., \& MCGinley, R. A. (1986). Hormones in saliva. Critical Review of Clinical Laboratory Science, 23, 95-146.

VINING, R. F., \& McGinley, R. A. (1987). The measurement of hormones in saliva: Possibilities and pitfalls. Journal of Steroid Biochemistry, 27, 81-96.

WADE, S. E. (1992a). Less-invasive measurement of tissue availability of hormones and drugs: Diffusion-sink sampling. Clinical Chemistry, 38, 1639-1644.

WADE, S. E. (1992b). An oral-diffusion-sink device for extended sampling of multiple steroid hormones from saliva. Clinical Chemistry, 38, 1878-1882.

WADE, S. E., \& HAEGELE, A. D. (1991). Time-integrated measurement of corticosteroids in saliva by oral diffusion sink technology. Clinical Chemistry, 37, 1166-1172.

Wilkins, J. N., Carson, H. E., van Vunakis, H., Hill, M. A., GRITZ, E., \& JARVIK, M. E. (1982). Nicotine from cigarette smoking increases circulating levels of cortisol, growth hormone, and prolactin in male chronic smokers. Psychopharmacology, 78, 305308.

Wolkowitz, O. M., Reus, V. I., Weingartner, H., Thompson, K., Breier, A., Doran, A., Rubinow, D., \& Pickar, D. (1990). Cognitive effects of corticosteroids. American Journal of Psychiatry, 147, 1297-1303.

(Manuscript received January 3, 1994; revision accepted for publication August 16, 1994.) 